\title{
TRABAJO SOCIAL ESCOLAR, CIUDADANIAA Y EDUCACIÓN: UNA REFLEXIÓN PERTINENTE
}

\section{Doris S. Pizarro Claudio ${ }^{1}$}

\section{Resumen}

El trabajo social escolar enfrenta el dilema de evaluar los paradigmas de la intervención social a raíz del deterioro de la educación pública. En la Carta Circular de Trabajo Social 16-2004-2005 del Departamento de Educación, predomina un discurso ambiguo y superficial, basado en enfoques positivistas de intervención, los cuales limitan la aportación que esta disciplina puede hacer al proceso educativo. En su praxis se privilegia la atención a casos en su modalidad individual o grupal, con poca atención al método comunitario. La atención del trabajo social a procesos de construcción de ciudadanía educativa, a la formación de liderazgo participativo de estudiantes, a familias y comunidad son estrategias lejanas en la mayoría de las escuelas. Los desaciertos en la descentralización y la invisibilidad de la autonomía educativa, constituyen barreras para la construcción de una nueva gramática en el trabajo social escolar. Sugiero reenfocar la intervención social desde una nueva relación con los demás componentes de la comunidad escolar y con su contexto macroestructural. Esta debe estar basada en la construcción de una ciudadanía educativa y en la revisión de las ideas sobre quiénes son los sujetos educativos.

Descriptores: Trabajo social escolar, ciudadanía educativa, sujeto educativo, autonomía educativa, participación escolar.

\section{Abstract}

School social work confronts a dilemma rooted in the deterioration of the public school system. Hence, this dilemma calls for the need to evaluate the paradigms of the school social work interventions. In the Department of Education's official memo for school social work \# 16-2004-2005, the language used is ambiguous and superficial. It is based on a positivism discourse about what the intervention should be, thus limiting what the social work discipline can bring to the educational process. This discourse in its

\footnotetext{
${ }^{1}$ Catedrática Auxiliar en la Escuela Graduada de Trabajo Social Beatriz Lassalle, Universidad de Puerto Rico en Río Piedras.
} 
practice focus the attention on individual casework and group work, thus limiting what the community work method could do to the educational process. The attention of school social work to the educational process of the social construction of citizenship, nurturing a participatory leadership among students, family and community is almost non-existence. The errors in the decentralization and the invisibility of the educative autonomy constitute barriers for the construction of a new grammar in the school social work. I suggest that the school social work should focus its intervention from a new perspective. It should be focused on a new relationship with all the members that constitutes the school community and within its macro structural context, based in the social construction of an educational citizenship and taking into consideration the ideas about educational subjects.

Key words: School social work, educational citizenship, educational autonomy, school participation.

\section{Introducción}

El propósito de este ensayo es dar una mirada crítica al Programa de Trabajo Social desde diversos enfoques epistemológicos, conceptuales, políticos y metodológicos. Para ello, me basé en contenidos de la Carta Circular de Trabajo Social Escolar (CCTSE) 16-2004-2005 (Departamento de Educación, 2004a) y en mi praxis como trabajadora social escolar. Examino las construcciones en torno a la intervención social, la construcción del sujeto, las familias y de la comunidad, así como la integración del trabajo social a los demás componentes del sistema educativo, sus concepciones filosóficas y la relación con su entorno. Me propongo problematizar estas construcciones desde la perspectiva de algunos conceptos de la teoría crítica: la concepción del Poder, el contexto socio- económico, los derechos educativos, el Estado, la ciudadanía y la intervención social.

De acuerdo a Guardiola (2006), la teoría crítica aborda la perspectiva de conflicto, así como la de transformación de las instituciones, las prácticas sociales y políticas. De igual forma reconoce que las relaciones de dominación se manifiestan como luchas sociales, las cuales se critican desde diferentes dimensiones, reconociendo las aportaciones de los actores sociales (Guardiola, 2006). La teoría crítica, además de censurar a la sociedad, también hace propuestas de cambio. Propongo evaluar la intervención social 
en el sistema educativo y examinar algunos retos de esta profesión a la luz del contexto en el cual se desarrolla.

\section{Construcción Histórica del Trabajo Social Escolar}

En Puerto Rico, la profesión de trabajo social se vinculó a la educación desde sus inicios en la década de 1920, con un proyecto del Departamento de Instrucción Pública dirigido al trabajo comunitario (Campos de Córdova y Córdova Campos, citado en Guardiola, 1998). El Programa de Trabajo Social Escolar (PTSE) se creó durante el curso escolar 1928-1929, conjuntamente con la formación de las primeras cinco Segundas Unidades Rurales. Para esa fecha también la "American Association of Social Workers" acreditó un Departamento de Trabajo Social, adscrito al Colegio de Pedagogía de la Universidad de Puerto Rico. Por su parte, Rivera de Alvarado (1986) ubica a la primera trabajadora social escolar- Guillermina Rivera de Antique- en la Escuela José Julián Acosta del Viejo San Juan para atender situaciones de familia y de ausentismo escolar. En los primeros años de la profesión, se reconocían dos tipos de trabajadores sociales: los(as) trabajadores sociales empíricos, quienes realizaban el trabajo social sin estudios formales en la disciplina; y por otro lado, los que tenían estudios formales cursados en Estados Unidos. Luego, a éstos se unieron los que lograban estudiar en la Universidad de Puerto Rico, cuando se institucionalizó la profesión (Rivera de Alvarado, 1986).

Más adelante, el PTSE se beneficiaría con la formación en 1954 del Programa de Maestría de la Universidad de Puerto Rico, aprobado por el Consejo de Educación Superior. Vale anotar la vinculación del trabajo social en Puerto Rico con las transformaciones macroestructurales y con la implantación de políticas sociales que se derivan de la imposición de los modelos coloniales de dependencia en el plano económico, cultural y social. Desde sus primeros momentos, la gestión del trabajo social se vinculó al apoyo que le prestaba la comunidad a la escuela. Esa función cambió con la transformación de una sociedad agraria a un modelo de desarrollo urbano, conjuntamente con el crecimiento del Departamento de Instrucción Pública (DIP). En 1960, se planteó la necesidad de crear en el DIP una división a cargo de prestar ayuda al estudiante (Claudio, citado en Cruz, 2006) a la cual se adscribió el Programa de Trabajo Social Escolar. Vale decir que en la Ley Orgánica del Departamento de Educación, apenas se menciona el Trabajo Social Escolar, el cual conforma el Equipo de Apoyo, conjuntamente con el 
Programa de Orientación y Consejería Escolar, Biblioteca y en algunos casos, el o la sicóloga escolar. A pesar de que la gestión de apoyo a la docencia se visualiza como una interdisciplinaria, se desconoce si en la práctica existe una coordinación y complementariedad adecuada en las escuelas o en otros niveles del sistema educativo. Considero, que en el ámbito escolar existe aún confusión en cuanto a los deberes y responsabilidades de cada profesión.

Actualmente hay una matrícula de más de 1,700 trabajadores y trabajadoras sociales ejerciendo en el DE. El sistema educativo es una de las dependencias gubernamentales de mayor empleo de trabajadores(as) sociales. Aún así, los enfoques de intervención del Trabajo Social son limitados para abordar la complejidad de la realidad escolar y de la intervención que se hace desde el PTSE en su relación con los demás componentes educativos. Una de las dificultades al profundizar en esta situación es que no existen muchas investigaciones sobre el Programa, lo que conlleva que se pierda la oportunidad de construir conocimientos basados en la praxis de trabajadores y trabajadoras sociales escolares. Cruz (2006) señala que ha existido inconsistencia en la gestión de supervisión del PTSE y que sólo dos Regiones Educativas han contado con supervisoras de trabajo social, mientras que en muchos distritos escolares apenas existe coordinación e intercambio entre las escuelas. En casos específicos, según me constadirectores y directoras no facilitan ese intercambio, basándose en la autonomía de las escuelas de la comunidad.

La Ley Orgánica del Departamento de Educación y el propio nivel gerencial del DE apenas toman en cuenta el PTSE y no se fomenta la sistematización de las intervenciones que se realizan. De acuerdo a Vélez Restrepo (2003), la evaluación, al igual que la sistematización son instancias importantes en el trabajo social para allegar nuevos conocimientos a la profesión. Sin embargo, el Programa no cuenta con criterios claros de calidad para medir la efectividad de la intervención social y mucho menos para sistematizarla. Son conocimientos que se pierden. Entre otras razones, ello se debe al volumen de situaciones en las cuales intervienen los trabajadores sociales, a que no se visualice la sistematización como parte de la intervención y a que no existe una cultura y apoyo para la labor de investigación. Las destrezas y las actitudes para la investigación no se enfatizan en el PTSE. De otra parte, los informes de labor cumplida se basan en estadísticas simples que no aportan a un conocimiento sobre la calidad de la intervención. 


\section{La Carta Circular de Trabajo Social (CCTSE)}

El Programa de Trabajo Social Escolar se regula por la Carta Circular 162004-2005 (2004a) aprobada por el Dr. Víctor Fajardo, en el 2000 y enmendada el 7 de octubre del 2004 por el Dr. César Rey, entonces Secretario de Educación. De acuerdo a lo estipulado por la CCTSE, el PTSE deberá prestar servicios de tratamiento y mejoramiento social a nivel individual y grupal a estudiantes, tutores(as) y comunidad. De igual manera, al PTSE se le asigna el establecer programas de educación y prevención dirigidos a toda la comunidad escolar, incluyendo el personal escolar. Además debe atender situaciones relacionadas con el clima escolar y al mismo tiempo, mantener una relación de coordinación y colaboración con otras agencias e instituciones que atienden el desarrollo de la niñez, la juventud y la familia.

En relación con los focos de atención, el trabajador o la trabajadora social deben identificar aquellas áreas o patologías que impiden el funcionamiento y el desarrollo social estudiantil (Departamento de Educación, 2004a). Considero que en la CCTSE se minimiza la atención hacia factores del clima escolar y hacia los procesos de implantación de políticas educativas. La Ley Orgánica de Educación (Ley 149) establece que el estudiante es el centro del proceso educativo, lo cual incide en el énfasis individual en los sistemas de intervención. El clima escolar, al igual que los procesos de políticas educativas, unidos al contexto socioeconómico y cultural de las comunidades de entorno son dimensiones que condicionan profundamente los procesos educativos, $\mathrm{y}$, por consiguiente, la intervención con individuos y grupos.

La CCTSE se vincula con las metas y los objetivos centrales del sistema educativo. De ahí que las necesidades priorizadas por el PTSE sean las situaciones que afectan el proceso educativo, entre éstas el desarrollo social de estudiantes, el abandono escolar y el ausentismo, la violencia en los planteles, problemas emocionales, situaciones familiares, así como el uso y abuso de sustancias controladas. En la medida en que se ha incrementado las situaciones de maltrato de menores, los embarazos en adolescentes, las enfermedades de transmisión sexual y la maternidad y paternidad de adolescentes, estos factores también figuran entre las prioridades del Programa. En el PTSE existe la tendencia marcada a focalizar y a diferenciar los grupos específicos de estudiantes (estudiantes sin hogar, jóvenes embarazadas, estudiantes en riesgo de uso de sustancias controladas) o por 
otras situaciones. Es una forma de fragmentar a la población estudiantil por características o condiciones particulares. $\mathrm{Al}$ tomar en cuenta estas necesidades, se observa que el trabajo social escolar aborda múltiples necesidades y situaciones que deben ser cubiertas también por otros niveles o agencias del Estado. Esto convida a que se trabaje con enfoques interdisciplinarios y con un conocimiento más integrado de las políticas públicas. En relación con ello, se han establecido diversos protocolos de colaboración interagencial, especialmente para el área de familia, salud, recreación, (entre otros), los cuales se incorporan como políticas del PTSE. Estos protocolos se diseñan a niveles superiores y luego bajan a las agencias.

Las metas y prioridades del Programa también se condicionan por las asignaciones de fondos federales contenidas en las políticas que se establecen en Estados Unidos y que se imponen a Puerto Rico en virtud de la condición colonial. Una de las políticas que más ha afectado al TSE,es la Ley "No Child Left Venid”. Por la vía de la imposición de las políticas federales, se promueven y se reproducen los enfoques de trabajo social de Estados Unidos, donde predomina el paradigma de la patología del individuo y de la sociedad, así como la invisibilidad de la lucha de clases y de la desigualdad social (Guardiola, 1998).

En la CCTSE apenas se menciona el ejercicio de los derechos civiles y sociales, la ciudadanía y la participación en la toma de decisiones como asuntos que también merecen atenderse con prioridad en el sistema educativo. stos factores inciden profundamente en el desarrollo personal del estudiantado y en el clima escolar. A raíz del movimiento de reconceptualización del trabajo social, iniciado en la década del setenta en América Latina, estos temas se visualizan como valores importantes de la intervención social. El movimiento de reconceptualización parte del reconocimiento de que el trabajo social opera como un mecanismo de control social, donde existe una contradicción entre las demandas de las instituciones y los sectores hacia quiénes se dirige (Guardiola, 1998). Esta orientación también plantea incongruencia entre las funciones del trabajo social tradicional y las expectativas de construcción de ciudadanía para un cambio social. 
El movimiento de reconceptualización ha estimulado a que se evalúe el papel del trabajador y la trabajadora social en las agencias estatales y en instituciones privadas. De igual modo, ha impulsado una proyección de la profesión entre lo macro y las prácticas particulares, por lo cual asume la democratización y el ejercicio de los derechos y de la ciudadanía como proyecto social (Alayón, 2005). En la mayoría de las escuelas, la participación y el ejercicio de los derechos de la comunidad escolar se encuentran en estado crítico, a pesar de que éstos forman parte del discurso de la mayoría de los documentos de políticas educativas. En las escuelas apenas se educa sobre las leyes y derechos educativos, según expresaron participantes de grupos focales realizados en el 2006 en una escuela de San Juan (Pizarro, 2007). Estos asuntos deben estar más acentuados en la agenda del Programa de Trabajo Social Escolar y en el Programa de Orientación y Consejería, y de igual modo, integrarse también en los currículos y en todas las dinámicas del proceso educativo. De igual manera, se deben integrar enfoques de ciudadanía, democracia, participación, derechos, conjuntamente con asuntos que se vinculan con la descentralización de las políticas sociales a raíz de las transformaciones ocurridas en tiempos de la globalización.

La construcción de una autonomía educativa, desde una perspectiva de integración de toda la comunidad escolar-tutores(as) estudiantes, líderes comunitarios y personal escolar-, debe formar parte también de la gestión de trabajo social escolar. La autonomía escolar implica dotar a la comunidad escolar de destrezas en el área de gerencia, administración, recursos humanos,- dimensiones que también forman parte de trabajo social. Pero la realidad es que la intervención social ha caído en la trampa de atender sólo las patologías sociales, construidas desde las visiones de los sectores de poder, sin adjudicar otros focos de intervención basados en fortalezas y más proactivos. En esa medida, se puede afirmar, que la intervención social en la escuela, se concentra más en atender síntomas de problemas sociales, que en crear contingencias o en prevenir los factores que los propician.

\section{Metas del Programa de Trabajo Social Escolar}

Las metas de trabajo social se vinculan con las prioridades del proceso educativo. De ahí que una de las metas contenidas en la CCTS (Departamento de Educación 2004a) se plantee de la siguiente manera: 
Contribuir al desarrollo de conocimientos y destrezas cognoscitivas y sociales que permitan al individuo comprender, disfrutar y mejorar la calidad de vida, ser capaz de tener una existencia personal y social plena en cada etapa de su vida, y de participar responsable y productivamente en la sociedad.

Para que esta participación social pueda darse, se requiere igualar la calidad educativa a la que acceden los diferentes grupos sociales (Calero y Bonal, 1999). En la actualidad, la mayoría de los estudiantes del sistema público provienen de sectores de pobreza. En Puerto Rico, los recursos asignados a las escuelas públicas dependen de los niveles socioeconómicos donde estén ubicadas. Las asignaciones de fondos se hacen desde una óptica residual y remediativa y no para transformar el deterioro de la educación pública. Con el deterioro de la calidad educativa, muchos estudiantes se han desplazado al sistema privado, buscando mayor seguridad y calidad educativa (Ladd \& Batiz, 2006). Otros han abandonado la escuela. Las asignaciones de fondos en el DE no necesariamente corresponde a la calidad de la enseñanza o la eficiencia de los programas. Ello depende de cómo se utilizan éstos, de la eficiencia con que se adjudica o se provee el servicio, así como de la participación de los sectores en la definición de necesidades y de prioridades.

Una de las críticas constantes a las escuelas públicas en sectores de escasos recursos es la falta de personal, la poca profundidad y pertinencia de los currículos y la falta de alternativas para el uso del tiempo libre. Existe una diferencia marcada en cuanto a los recursos que se asignan a las escuelas especializadas y los que se asignan a planteles regulares localizados en áreas pobres. Pocos estudiantes de comunidades de bajos ingresos, barriadas o residenciales tienen acceso a escuelas especializadas, ya que los requisitos de admisión son altos y los costos de materiales para permanecen en éstas a veces resultan prohibitivos para familias pobres. En esas condiciones, contribuir a transformar los enfoques excluyentes y las condiciones materiales de las escuelas, al igual que la calidad del proceso educativo debe figurar en las metas del trabajo social escolar como requisito para mejorar la calidad de vida de estudiantes y aportar responsable y productivamente a la sociedad, como dice la CCTSE. 


\section{Educación como control y reproducción de los sistemas dominantes}

Gil (1998) señala que la educación es una de los mecanismos para perpetuar las formas del trabajo en el sistema capitalista. A través de ésta, se logra que la niñez y la juventud se socialicen en las formas que ocuparán en el futuro en el mundo del trabajo como adultos. En Puerto Rico hemos visto ese rol de cooptación por parte de Estados Unidos a partir de la invasión del 1898, cuando la educación se convirtió en mecanismo de asimilación y de colonialidad (Negrón de Montilla, 1970). Por lo tanto, el Programa de Trabajo Social debe interceder también por una educación descolonizadora integrada al conocimiento de los derechos y la autodeterminación y que tenga como objetivo el fortalecimiento de las raíces culturales nacionales.

$\mathrm{Al}$ igual que en otros escenarios de trabajo social, el o la trabajadora social se constituye en un representante o mediador del Estado en relación a las políticas sociales que ejecuta. Guardiola (1998) advierte que en muchas ocasiones ello va en contra de los intereses de los y las participantes de los programas. En ese momento surge el reto de trabajadores y trabajadoras sociales en relación a su ética. De ahí que haya que profundizar en esta construcción del trabajo social que subyace en la CCTSE. De acuerdo a la Carta Circular de Trabajo Social (Departamento de Educación, 2004a), “el trabajador social es un profesional que ayuda a individuos, familias, grupos y comunidades a enriquecer, fortalecer y restaurar su capacidad para el funcionamiento social y crear las condiciones sociales que contribuyen a ese propósito".

Siguiendo el planteamiento de Torres (2001), -para poder prestar un servicio adecuado - el trabajo social escolar debería entender sobre las teorías del Estado y su relación con la educación. O lo que el mismo autor define como pensar en la educación y el trabajo social en términos políticos. Ello conllevaría a que el Programa de Trabajo Social del Departamento de Educación entienda las contradicciones a las cuales se enfrenta el sistema educativo en Puerto Rico en su relación con el Estado colonial. Entre los fenómenos relacionados que impactan, a la educación, se encuentran el colonialismo y dependencia, la pobreza y marginalidad; la distancia a la tradición y a la historia. De igual forma inciden la falta de visión sobre la raíz de los problemas que afectan a la educación. Este planteamiento surge a la luz del interés de la política pública que establece la Ley "No Child Left 
Behind", la cual aspira a la estandarización de conocimientos, la estigmatización del alumnado y de sus familiares en un sistema donde lo que predomina es la meritocracia en un marco de desigualdad y de exclusión de oportunidades.

Conviene en ese sentido, a la hora de examinar las metas del PTSE, anotar el planteamiento de Torres (2001) en relación a la vinculación de las teorías del Estado y las políticas educativas. Los enfoques conceptuales sobre esta relación condicionan las posturas del trabajador(a) social. Torres plantea que las teorías del Estado dan sustancia a los planteamientos éticos y morales y a los papeles atribuidos a la educación en la socialización y construcción cognoscitivas de las identidades culturales. La vinculación que hace el estado entre poder, estado y sociedad guían la constitución de políticas educativas. De igual modo, condicionan la implantación de las políticas por los diversos componentes de la comunidad escolar, incluyendo el equipo de apoyo a la labor docente, donde se adscribe el trabajo social.

Uno de los objetivos del PTSE, de acuerdo a su Carta Circular es: "desarrollar un servicio de trabajo social que facilite la prevención, identificación temprana, reducción y manejo adecuado de situaciones que inciden negativamente en el funcionamiento escolar y social de los estudiantes". Este objetivo puede tener diversas lecturas. ntre las situaciones que pueden incidir negativamente en el funcionamiento escolar y social de estudiantes, pienso en actores de pobreza, exclusión, desigualdad en servicios educativos, ituaciones de violencia y maltrato institucionalizado, entre otras situaciones que caracterizan el clima escolar. stán las condiciones físicas o factores emocionales que se agravan o se inhiben por la falta de apoyos efectivos. En Puerto Rico existe un abandono y alta de reconocimiento al ejercicio de los derechos civiles; y de igual manera, existen fisuras en los procesos democráticos. Otras condiciones que afectan el funcionamiento de estudiantes son el discrimen en la prestación y en la calidad de los servicios educativos, los currículos impropios y la poca participación de estudiantes y sus familias en los procesos pedagógicos. Las relaciones inadecuadas de muchos estudiantes en el seno familiar o con el personal escolar, al igual que factores estresantes de la comunidad inciden en el funcionamiento escolar.

En los grupos focales que realicé para mi disertación doctoral, alumnos y alumnas, tutores(as) y líderes de la comunidad criticaban la poca o ninguna 
participación en la toma de decisiones en la institución escolar como una situación que les afectaba material y subjetivamente. Omitir oportunidades e imponer reglas sin participación son situaciones que pueden generar violencia escolar. También los medios y el mercado crean expectativas sociales que afectan el comportamiento de las personas y los grupos. Los factores de opresión institucionalizada nciden negativamente en el funcionamiento escolar y social (Barreto y Quiñones, 2000, Young, 1998). La ausencia de derechos y ciudadanía, la desigual distribución de poder y decisión en cuanto a lo que se enseña, cómo se enseña y las sanciones injustas dentro del sistema escolar, crean tensión en la comunidad escolar. Las violaciones a los derechos humanos y ciudadanía implican intranquilidad social, conflictos y violencia, según la Carta de Derechos (Organización de Naciones Unidas, s.f.). Prevenir la violencia escolar y fomentar un clima de convivencia figuran entre las metas del PTSE. Entre los derechos educativos que contribuyen a la convivencia figuran el derecho de expresión, asociación, a ser informados sobre evaluaciones, religión, afiliación política, así como el derecho a la presunción de inocencia y a no ser sometidos(as) a sanciones injustas. La escuela debe garantizar los derechos a estudiantes y familias ya que los menores poseen también un estatus de ciudadano(a).

Van Soest (1997) establece tres tipos de violencia y los vincula con el ejercicio de los derechos humanos. Una forma de violencia es que implica la omisión de brindar servicios que necesita la persona para su desarrollo. La autora también tipifica como violencia por represión la infracción a los derechos políticos, económicos y sociales. La tercera forma de violencia es la violencia por represión, que es impedir el desarrollo de las personas a su autoestima, a su dignidad y a su bienestar social. Por su parte, Mc Afee (citado por Van Soest, 1997) define el concepto de desarrollo, como el proceso que da autonomía y autosuficiencia a las personas para ir más allá de las ayudas y la emergencia, que les permite ser menos dependientes y vulnerables a los desastres, independientemente del género y la cultura. Estos principios establecidos por Van Soest se violan en el sistema educativo al no facilitar el empoderamiento y la autogestión de los componentes de la comunidad escolar a través de la participación y de otras formas de construcción de procesos de ciudadanía.

Al tomar en cuenta el carácter histórico del trabajo social, trasciende que esta disciplina no puede ejercerse descontextualizado o atemporalizado (Vélez Restrepo, 2003). El carácter histórico de la intervención social, me lleva 
también a subrayar el impacto que ha tenido la globalización y las políticas neoliberales en el ejercicio de esta profesión ya que la CCTSE vigente se redactó desde la perspectiva de la globalización y del neoliberalismo, con las consecuencias correspondientes.

\section{El impacto de la globalización en el trabajo social escolar}

Una de las complejidades del trabajo social es lidiar con las contradicciones que resultan de la desigualdad del entorno y de las opciones del participanteen este caso el estudiante, sus familias y la comunidad - para lidiar con éstas. En la globalización estas contradicciones se agudizan y se representan en situaciones de pobreza, pocas oportunidades de desarrollo y situaciones de marginalidad. Brown (citado en Ahearn (2003), dice que la globalización es una comprensión del mundo como un todo, en el cual se ubican acontecimientos desde lo local hasta lo global. Esta aseveración de Brown retrata una contradicción del sistema educativo de Puerto Rico donde las políticas públicas federales predominan sobre los intereses nacionales, aunque contengan realidades ajenas a las nuestras. Así mismo, los contenidos educativos y sus estándares resultan muchas veces ajenos y poco pertinentes a los grupos estudiantiles y sus comunidades.

La globalización conlleva una reconfiguración del Poder, un mayor dominio de los intereses económicos y sociales del mercado, protegidos a su vez por un Estado Benefactor con menos poder. Ese dominio del capital surge en la medida en que se ha ido desmantelando el Rol del Estado Benefactor y éste disminuye su capacidad para la gestión pública. En Puerto Rico, el estado colonial ha sido incapaz de adaptar la educación a las necesidades socioeconómicas y políticas del país y actuar sobre ellas con eficiencia, como acontece con la educación pública.Una de las consecuencias de esto es que se ha lesionado los derechos en el sistema educativo. A raíz de la implantación de la Ley 149 la descentralización y la llamada autonomía de las escuelas han facilitado la privatización de áreas de la educación que antes se prestaban desde el propio DE. Lo anterior conlleva a que ahora, la intervención social, no dependa sólo del Equipo de Apoyo a la Docencia, pues para ello se subcontratan otros profesionales. lgunas de las modalidades de la privatización conllevan la falta de seguimiento y el desconocimiento de los alcances de la intervención por la falta de monitoría, evaluación de resultados y de sistematización. 
El fenómeno de la globalización y el neoliberalismo también ha impactado a las subjetividades y la identidad de los componentes de la comunidad escolar en la medida en que define a la sociedad civil en términos del mercado (García Canclini, 1995). Se cambia el concepto de ciudadano al de consumidor(a) o cliente y se da el cambio en los patrones de identidad a la ideología del consumo, con la influencia marcada de los medios y de la informática. Todo esto ha conllevado a que la educación transforme los sentidos que se le ha adjudicado tradicionalmente. Ya no es la ideología o el paradigma del trabajo o el progreso lo que predomina en los significados educativos. La educación ya no constituye la llave para alcanzar un empleo o accesar mejores condiciones de vida. A ese cuadro se añaden los factores de opresión que se refuerzan con el deterioro de las políticas públicas: el racismo, sexismo, xenofobia y desigualdad de clases y falta de reconocimiento de la diversidad. Estos factores multiculturales son espacios de lucha y organización para la disciplina de trabajo social en las escuelas.

La globalización ha traído un desplazamiento de la comunidad o la localidad como el centro de referencia de los grupos sociales (Ahearn, 2003). Ahora los centros se enmarcan en los medios masivos de comunicación, en la medida en que se destruyen las instituciones locales por sistemas sociales, económicos y políticos externos. La cultura está bajo una fuerte influencia extracomunitaria, como son los medios de comunicación, la música, las artes, el ambiente (Ahearn, 2003). Hay nuevas nociones para involucrar a los ciudadanos en los asuntos sociales. El reto es cómo integrar estos nuevos fenómenos sociales a la gestión educativa y a la gestión del trabajo social escolar para reforzar la autonomía crítica y la reflexibilidad de los sujetos educativos.

\section{Construcción del Trabajo Social}

Con estos planteamientos sugiero una reconceptualización de los paradigmas que orientan el trabajo social escolar. No sólo a partir del discurso de la Carta Circular (Departamento de Educación, 2004a), sino también desde la praxis, la ética y las visiones epistemológicas que le orientan. Detrás de las políticas públicas y de los programas subyace una forma de ver el mundo y de interpretar la realidad (Matus, 2003). La CCTSE se ubica en una dimensión de sistemas, donde el trabajo social conforma uno de los subsistemas de 
apoyo del sistema educativo. Sin embargo, esa posición es limitada en la medida en que no crea contingencias adecuadas para abordar la complejidad que supone la relación entre sus diferentes componentes, especialmente los que se derivan del nivel macroestructural.

La dominación colonial y el fenómeno de la dependencia y la asimilación cultural impactan los enfoques de las políticas educativas en Puerto Rico. Guardiola (1998) destaca que en las políticas sociales ha predominado el asistencialismo y los enfoques residuales y redistributivos. Estos enfoques no van dirigidos al desarrollo y a la autogestión de las personas y de los grupos. La fragmentación es otra de las características del sistema educativo que le impide mantener los sistemas funcionando. Eliminar la fragmentación, conllevaría el facilitar la integración de tutores a la toma de decisiones, compartir información, distribuir el poder entre otras necesidades del sistema, crear nuevas visiones de gerencia escolar, seleccionar y evaluar el personal y decidir sobre la utilización del presupuesto. Se pretende que el sistema continúe funcionando de manera jerárquica y cerrada, donde la energía no se intercambia de un sistema a otro. En los grupos focales que realicé en la Escuela República del Perú se destacó el interés de tutores y estudiantes de participar con sentido en decisiones de presupuesto, organización escolar y en la selección de programas. De otra parte, se encontró que el personal docente subestimaba el alcance de esa participación.

Las visiones del trabajo social escolar no se distancian de los enfoques funcionalistas y de las visiones de costo beneficio que imperan en la administración pública. De acuerdo al Departamento de Educación (2004a), "el trabajador social dirige su atención prioritaria para atender las condiciones de salud, enfermedades mentales, ducación, pobreza, delincuencia, desamparo y deficiencias sociales o ambientales que inciden sobre los individuos o grupos que atiende". Vemos en esta construcción que el sistema educativo enmarca el trabajo social en un plano empírico, funcionalista, lo cual se refleja en los procesos de la intervención. El o la trabajadora social está llamada a reducir los indicadores de disfunción del individuo y su grupo, lo que al final de cada año se refleja en informes estadísticos que esconden la complejidad y las contradicciones de la existencia social de estudiantes, sus familias y la comunidad en su relación con su ambiente y otros factores macroestructurales. 
Uno de los desafíos que debe enfrentar trabajo social para ejercer sus funciones desde nuevos paradigmas, es identificar cuáles son las raíces de los problemas que afectan a la comunidad escolar y su existencia social. Esas situaciones, en la mayoría de los casos, están vinculadas con los factores de pobreza y de opresión institucionalizada, los cuales evitan que las personas se desarrollen y ejerzan todos los atributos de la ciudadanía educativa. Veo en muchos casos que a estudiantes y a tutores se les inferiorizan o se les discrimina por condiciones de vida, nivel educativo o formas culturales. Cuando esto sucede, no se les reconoce autonomía y se desoyen las críticas que éstos hacen al sistema. Sin embargo, la Carta Circular (Departamento de Educación, 2004a) establece que "Estos profesionales enriquecen la habilidad del sistema educativo para lograr su misión académica, especialmente en lo que respecta a la colaboración necesaria entre los padres, la escuela y la comunidad".

Trabajadores y trabajadoras sociales podemos hacer una aportación para enriquecer el sistema educativo con la participación de padres, madres, estudiantes y otros sectores de la sociedad civil interesados en aportar a la educación. Dicha gestión conlleva contribuir a la integración del sistema educativo, a fomentar redes entre la escuela y la comunidad y a trascender la colaboración, dar opinión o la consulta como las formas primordiales de participación. También podemos aportar forjando reciprocidad y solidaridad entre los diferentes subsistemas escolares y comunitarios. Esto conlleva el contribuir a la formación de un nuevo liderazgo basado en la participación, en la toma de decisiones educativas de estudiantes, tutores y tutoras, la comunidad y el personal, según dicta la política pública, específicamente la Ley Orgánica del Departamento de Educación (Ley 149) y la Carta Circular de Padres (Departamento de Educación, 2006). De hecho, una recomendación inmediata es deconstruir los discursos y los significados de la participación que se promueven en la Carta Circular para comprender cómo se están implantando y si se fomenta desde una visión de horizontalidad.

El trabajo social tiene atributos de cuidar, tratar y proteger (Epstein, 1999). La construcción histórica de esta profesión ha fortalecido los estándares generalizados por el Estado y por las instituciones dominantes sobre las conductas personales y estados subjetivos, a la par que también ha usado su poder para controlar el pensamiento y la conducta de personas e instituciones (Epstein, 1999). La trabajadora y el trabajador social se encuentra en una 
posición de Poder y de autoridad frente a los participantes de los programas (Guardiola, 1998), especialmente con estudiantes y sus familias, quienes en muchos casos carecen de organización y tienen mayor dispersión como grupo al reclamar servicios o al tratar de incidir en las políticas educativas. Esta desigualdad en la distribución de poder afecta la subjetividad y la identidad de estudiantes, tutores(as) y se convierten en barreras para el desarrollo de la autonomía de estos grupos. Las investigaciones sobre la participación de tutores en las escuelas señalan que el personal escolar ve con suspicacia la participación de tutores, la comunidad y los estudiantes; mientras simultáneamente, los (as) tutores (as) se sienten intimidados(as) al reclamar sus derechos educativos (Bedwell, 2004; De la Guardia \& otros, 2002; Fine, 2000). Un reto de profesionales del trabajo social, independientemente del escenario en el cual se desempeñen, es incidir sobre los discursos que estigmatizan y oprimen a los grupos de menos poder.

En su discurso, la CCTSE pone énfasis en la prevención, en la investigación social, trabajo con los padres y con la comunidad, la colaboración en las tareas del Director al igual que en la rendición de cuentas al Distrito Escolar. También reconoce la necesidad de formación constante de los trabajadores y trabajadoras sociales. Cónsono con la Ley Orgánica de Educación, la CCTSE pone énfasis en la intervención directa con los estudiantes, a quienes se les denomina el centro del sistema educativo. En la práctica profesional, también se privilegia el énfasis en la atención a casos. La intervención se focaliza en la atención a personas o grupos, y se minimiza la gestión para transformar estructuras, clima escolar, factores subjetivos que inciden en las relaciones de poder entre los diferentes grupos escolares. La atención a casos limita el que la intervención social en el ámbito escolar pueda utilizar también los enfoques comunitarios para ampliar los espacios y las metodologías de intervención social. El deterioro del sistema educativo, la descentralización de las políticas educativas y la construcción de una verdadera autonomía escolar requiere de la movilización y la organización de la comunidad y de otros sectores de la sociedad civil. Requiere el implantar procesos de planificación donde la escuela se vincule a las necesidades de la comunidad y, al mismo tiempo, promueva la acción social y la presión de ésta para los reclamos educativos al Estado. De esa manera, el trabajo social escolar puede ser un intercesor y facilitador para fortalecer instancias de la sociedad civil. 
De otra parte, la alta cantidad de casos que se refieren al PTSE, unidos a múltiples eventos cotidianos que requieren la atención urgente de trabajadores y trabajadoras sociales, pone en riesgo la calidad de la intervención. Esto se agrava por el seguimiento de procesos administrativos que requieren algunos programas-como el de Educación Especial- y las obligaciones que surgen de algunas políticas federales. Una de las limitaciones del Programa es que no cuenta con presupuesto asignado para realizar sus funciones, ya sea a nivel central como a nivel de una escuela. Tampoco se le asignan recursos administrativos ni tecnológicos. Para realizar sus funciones $\mathrm{y}$ atender otras iniciativas, las trabajadoras sociales allegan fondos a través de propuestas, donativos o actividades internas. De esa manera, el trabajo social innovador en alguna escuela queda subordinado a las posibilidades de cada incumbente - según lo permitan las tareas, la filantropía, o la habilidad para preparar propuestas para allegar fondos. La falta de recursos puede limitar el trabajo social a la intervención de casos.

\section{Construcción de los Sujetos}

La CCTSE reconoce que la labor educativa es un esfuerzo colaborativo de todos los componentes educativos. Sin embargo, la transformación de los males sociales- operacionalizada en la metodología de caso- pone énfasis en el o la trabajadora social y no en la capacidad de los propios sujetos, en su interacción con los demás grupos- para transformar esas realidades. Un elemento necesario para la transformación social sería que el sistema educativo tuviera de referencia un proyecto social con una filosofía donde la educación constituyera un mecanismo de cambio social y económico, del pensamiento crítico, basados en una pedagogía para la liberación, construida con la participación de todos los grupos (Freire,1970). Una educación basada en un proyecto social tendría mayor sentido para toda la comunidad escolar, especialmente para estudiantes, familias y comunidades. Considero, que el trabajo social escolar puede aportar a la búsqueda de ese horizonte perdido. El reconocimiento de otros componentes de ciudadanía, como son los derechos democráticos, el sentido de pertenencia, la autonomía crítica, al igual que la participación en el reclamo de servicios eficientes de educación, la autogestión y el empoderamiento son referentes que se pueden sumar al trabajo social escolar.

En relación al sujeto educativo, la Carta Circular (Departamento de Educación, 2004a) señala lo siguiente: 
El Programa de Trabajo Social Escolar aspira a la formación integral mediante la atención y prevención de situaciones que afectan su desarrollo óptimo. Fomenta su participación en diversas actividades educativas y sociales dirigidas a fortalecer el desarrollo de sus aspectos físicos, emocionales e intelectuales, para que mejore su calidad de vida, y desarrolle los principios necesarios para contribuir a la sana convivencia, a la justicia y a la paz social.

Para lograr la meta de un sujeto educativo que acceda a una calidad de vida superior y que contribuya a la sana convivencia como aspira la CCTSE, el trabajo social debe estimular la reflexibilidad del sujeto, fortalecer su autonomía (Matus, 2003), y facilitar una participación de calidad en la toma de decisiones relacionadas con actividades y acciones que se dan en el ámbito escolar. Es importante que los sujetos tengan la posibilidad de incidir en la transformación de su realidad mediante el análisis y solución de los problemas que le afecten, lo cual son atributos de la ciudadanía. El Estado capitalista moderno normaliza la ciudadanía y organiza a las personas para constituir la mano de obra pública y privada en el mercado del trabajo, que mantiene la economía y el orden social ya sea por coerción o por consenso (Epstein, 1999). Estas son funciones que se instrumentan a través de las políticas educativas. Las ideas que se construyan sobre los sujetos y sobre la educación condicionan las relaciones que se fomentan entre los grupos escolares y la sociedad. En el sistema educativo, el trabajo social también ejerce un control y autoridad sobre sus participantes: estudiantes, familias, comunidad y los normaliza, visto desde la perspectiva de poder de Foucault.

La misión de un Programa descansa en su dimensión conceptual y epistemológica (Matus, 2003). Cabe cuestionar qué paradigmas predominan en el Programa de Trabajo Social Escolar y en el sistema educativo puertorriqueño al cual se adscribe. En la sociología de la educación se han creado nuevos enfoques en cuanto a la realidad de los sujetos (Torres, 2001). Torres plantea que la educación se aleja del paradigma positivista y que se plantean nuevos paradigmas culturales. El referido autor señala que se debe tomar en cuenta las relaciones y las subjetividades de los grupos escolares, impactadas por el Poder institucionalizado. Sin embargo, los planteamientos de Torres no están presentes en los paradigmas del trabajo 
social, aun cuando sabemos que as escuelas también son espacios de conflictos por discrimen por género, raza, orientación sexual o nacionalidad. En las políticas educativas existe un doble discurso. Se aprueban políticas basadas en el discurso de participación, pero las iniciativas participativas se mantienen en bajo perfil. Se habla e autonomía educativa, pero se institucionalizan medidas de mayor control y poca flexibilidad en la organización escolar y en la implantación de programas. También destacan las medidas de privatización que favorecen al mercado, al igual que la eliminación de derechos laborales el personal educativo. Hay menos democracia y más elitismo en el proceso educativo, lo cual se agrava con la imposición de estándares en los currículos. Los enfoques funcionalistas impuestos a Puerto Rico desde la esfera federal no toman en cuenta los contextos en los cuales se desarrolla el proceso educativo, a la par que estigmatizan a las poblaciones escolares que no alcanzan los estándares. Estas situaciones condicionan también la metodología de la intervención, la selección de programas y la autonomía para asignar recursos a la intervención social.

Los señalamientos de los párrafos anteriores están relacionados con la responsabilidad primaria que se asigna a trabajo social, que es identificar a los estudiantes con necesidad de intervención social. Esta responsabilidad que se asigna a trabajadores y trabajadoras sociales parte de una visión de política social residual. Se buscan sujetos desvalidos, con alguna patología, con falta de motivación hacia el progreso escolar y con poca disposición para trascender en circunstancias de adversidad. Es una imagen inferiorizada de los sujetos educativos, incapaces de convertirse en sujetos de cambio ante el deterioro del proyecto educativo que sostuvo el modelo de dependencia, junto con las políticas asistencialistas del Estado Benefactor. El reto ético de trabajadores y trabajadoras sociales escolares consiste en desafiar la decisión de intervenir sobre síntomas, para intervenir sobre los fenómenos que verdaderamente afectan la convivencia y el desarrollo; es decir; en la interacción del fenómeno y el potencial de los sujetos para hacer reclamos educativos. Ello apela a la dimensión política del trabajo social.

Matus (2003) señala que estudiar los modelos de intervención social que se realicen y sus formas enunciativas resulta clave en el logro de mayores oportunidades para el desarrollo y fortalecimiento de la ciudadanía. De acuerdo a la profesora chilena, la intervención social se basa en una comprensión compleja y diferenciada de lo social. Vemos que en la Carta 
Circular de Trabajo Social lo social se simplifica enumerando síntomas. Todo programa debe ser evaluado desde la óptica de la intervención social y no sólo como formas de operacionalizar políticas sino también como gestión de fortalecimiento de espacios públicos (Matus, 2003). En este caso, veo que el trabajo social escolar puede contribuir a fortalecer el proceso educativo, fomentando componentes de ciudadanía en el ámbito escolar desde la niñez y la adolescencia. Entre estos componentes figuran el ejercicio de los derechos, la justicia social y la equidad, el reconocimiento de la diversidad, la participación y toma de decisiones, la promoción de redes y alianzas educativas, entre otros atributos ya mencionados.

El trabajo social también debe permitir abordar la cuestión cultural coherentemente, ya que en ellos también se perfilan fenómenos sociales que podemos intervenir (Matus, 2003). Lo cultural provee nuevas formas de intervención social partiendo del reconocimiento de la diversidad entre los grupos escolares y comunitarios. Es hacia esos fines que Fine (2000) señala que la participación de tutores en procesos educativos no es representar a su progenie, sino aportar su cultura y la de sus comunidades. En esa dirección, sugiero se analice a profundidad los contenidos de la Carta Circular (Departamento de Educación, 2006) sobre la Participación de los padres y madres.

Desde el trabajo social se construyen diversas visiones sobre los sujetos escolares. Una que llama la atención es la victimación de los grupos e individuos, la cual les resta autonomía (Matus, 2003). La visión de un otro subordinado, construye imaginarios socioculturales: estudiante violento, madre dependiente, familias inoperantes, fulano indisciplinado que sirven para etiquetar y estigmatizar a los grupos (Matus, 2003). Sobre estas etiquetas se justifican procesos de exclusión y marginación, como llevarse a excursiones sólo a los 'buenos' mostrar preferencia por tutores de mayor nivel educativo. Se parte además de referentes de valores y una ética bien permeada por el Poder y la autoridad dentro del sistema educativo de una sociedad ya de por sí diferenciada. En esa medida se reduce y se desvalora el fundamento de la ciudadanía y de los derechos - en este caso de la comunidad escolar.

Considero también de valor para esta reflexión, el señalamiento de Matus (2003) sobre la tendencia de trabajadoras y trabajadores sociales a guiarnos por una compulsión a la acción (empiria) respondiendo a eventos y a 
urgencias cotidianas en detrimento de un conocimiento más profundo y una perspectiva analítica y crítica de comprensión del fenómeno sobre el cual se desea intervenir. Esto sucede con el fenómeno de la violencia escolar, limitado a veces a la observación del número de peleas entre estudiantes, sin abordar los factores de opresión institucionalizada que domina en los planteles y que merece atención profunda. Por ejemplo, maestros o maestras envían listas de estudiantes, con una marca ya puesta sobre su 'disfuncionamiento', encajonados en 4-6 indicadores conductuales y empíricos. Se espera, que se tome la lista y se intervenga desde la racionalidad positivista para disminuir o eliminar el 'disfuncionamiento'. Los niveles de efectividad de la intervención y los informes estadísticos se equiparan a la cantidad de personas que pasaron por la oficina.

En más de una ocasión trabajadores y trabajadoras sociales, al igual que consejeros y consejeras estudiantiles han señalado la falta de espacio y tiempo para dar seguimiento y profundizar en la calidad de las intervenciones sociales. En muchos casos se requiere un esfuerzo multidisciplinario que no se facilita en el sistema educativo, bien por el deterioro del sistema, la falta de recursos o por la falta de reciprocidad y solidaridad de los componentes educativos. La situación se agrava también por la indiferencia del Estado para atender las desigualdades sociales y su pérdida de poder para supervisar el acceso y la calidad de los servicios privatizados por el mercado.

Una aportación que puede hacer trabajo social escolar es dar cuenta de cómo fenómenos como la pobreza, la marginación, la exclusión que son dimensiones relacionadas con el Poder, afectan los resultados educativos y el desarrollo social del estudiantado. Halsey (1997) apunta que la educación siempre ha sido un foco de lucha entre aquellos con el Poder de decidir lo que es el conocimiento, $y$ aquellos excluidos de la toma de decisiones en la educación. El trabajo social, al igual que la educación debe ser considerado como una propuesta que permita la apropiación y la transformación por los participantes de su mundo material a partir de los procesos pedagógicos.

\section{A modo de resumen}

En estas reflexiones no se abordaron todos los aspectos de la CCTSE y del PTSE. Quise hacer una reflexión siguiendo un mapa que incluyera el contexto, lecturas diferentes sobre el contenido de la CCTSE, construcciones sobre el trabajo social y la comunidad escolar que inciden en los procesos de 
intervención. También abordé algunas ideas sobre el sujeto educativo, así como la vinculación del trabajo social con otros componentes del sistema que merecen evaluarse.

Otros temas que ameritan atención son la metodología y los modelos de intervención promovidos por la CCTSE, las relaciones con figuras escolares de autoridad así como la coordinación con otras disciplinas de apoyo a la gestión educativa. A este respecto, sugiero que se realicen investigaciones desde paradigmas construccionistas para profundizar en los significados, alcances, conceptos y metodologías del Programa desde los diversos actores educativos.

\section{Referencias}

Ahearn, Frederick L. (2003). lobalización: Implicaciones para la participación ciudadana. Revista de Trabajo Social. Pontificia Universidad Católica de Chile, 71, 7-26.

Alayón, Norberto. (2005). Trabajo social latinoamericano: A 40 años de la reconceptualización. Argentina: Espacio Editorial.

Barreto Cortéz, Esterla. \& Quiñones Rosado, Raúl. (2000). Un modelo de bienestar y desarrollo integral y sus implicaciones para la profesional de ayuda. Análisis, 2, 1-30.

Bedwell Rodríguez, George. (2004). Participación de los padres, madres, apoderados y apoderadas en el ámbito educativo: Una mirada de los dirigentes $y$ dirigentas de centros de padres y apoderados/as. Universidad de Santiago de Chile. Recuperado el 23 de agosto de 2005, de http://www.cybertesis.cl/tesis/chile/2004/bedwell9/doc/bedw

Bustelo, Eduardo. (1998). Expansión de la ciudadanía y construcción democrática. En Eduardo Bustelo \& Alberto. Minujin (Eds.), Todos entran: Propuesta para sociedades incluyentes (pp. 237-277). UNICEF: Colección Cuadernos de Debate.

Calero, Jorge; Bonal, Xavier. (1999). Política educativa y gasto público en educación: Aspectos teóricos y una aplicación al caso español. Barcelona: España: Ed. Pomares-Corredor. 
Cruz Santiago, Edda. (2007) Política y práctica de la supervisión del trabajo social en Puerto Rico: Lineamientos para construir propuestas alternas. Revista Hologramática. Facultad de Ciencias Sociales - UNLZ - Año IV, Número 6, V4 (2007), pp. 67 -91. Accesado en www.hologramatica.com.ar o www.unlz.edu.ar/sociales/hologramatica el $19 / 11 / 07$.

De la Guardia Romero, Rosa M.; \& Rosales Alamo, Manuel José. (2002). Concept de participation educative des enseignants et des parents. Universidad de La Laguna, Tenerife, Islas Canarias. Recuperado el 25 de enero de 2006 de http://absysweb.ccti.ull.es/cgi/-bin/abwebp.exe/li/ti

Departamento de Educación (2004a). Carta Circular de Trabajo Social 162004-2005. Normas para la Organización y el Funcionamiento del Programa de Trabajo Social Escolar. Puerto Rico: Autor.

Departamento de Educación. (2004b). Reglamento General de Estudiantes del Sistema de Educación Pública de Puerto Rico. San Juan: Autor.

Departamento de Educación. (2006). Carta Circular 18-2006-2007. Política Pública sobre Participación de Padres...en la Escuela. Puerto Rico: Autor.

Duschatzky, Silvia. (2000). Tutelados y asistidos: Programas sociales, políticas públicas y subjetividades. Buenos Aires, Argentina: Editorial Paidós.

Epstein, Laura. (1999). The culture of social work. En Adrenne Chambon, Allan Irving y Laura Epstein (Eds), Reading Foucault for social work, 3-27. New York: Columbia University Press.

Fine, Michelle. (2000). Parent involvement: Reflections on parents, power, and urban public schools. Recuperado el 15 de marzo 2006 de $\mathrm{http}: / / \mathrm{www} . \mathrm{tcrecord}$.org

Freire, Paulo. (1970). Pedagogía del oprimido, 1ra ed. Madrid, España: Siglo XXI. 
García Canclini, Néstor. (1995). Consumidores del siglo XXI: Ciudadanos del siglo XVIII: Conflictos multiculturales de la Globalización. México: Grijalbo.

Gil, David. (1998). Confronting injustice and oppression. New York: Colombia University Press.

Guardiola Ortiz, Dagmar. (Ed) (2006). El trabajo social en el Caribe hispano antillano: El contexto regional: una perspectiva crítica comparativa. Vol 1. Río Piedras: Editorial. Universidad de Puerto Rico.

Guardiola Ortiz, Dagmar. (1998). Trabajo Social en Puerto Rico: ¿Asistencia, Desarrollo, Transformación. Puerto Rico: Editorial Edil.

Halsey, Albert; Lauder, Hugh;.Brown, Phillip; Stuart, Amy. ( 1997) (Eds.). Education, culture, economy and society. Londres: Oxford University Press.

Ley Orgánica del Departamento de Educación. (1999).

Ley No Child Left Behind (1999).

Ladd, Helen F.; \& Rivera Batiz, Francisco. (2006). Education and economic development. En Susan Collins, B. Bosworth \& Miguel Soto Class (Eds.), Restoring growth in Puerto Rico: Overview and policy options (pp. 43-55). Washington, DC: Brookings Institutional Press.

Matus, Teresa. (2003). La Intervención social como gramática: Hacia una semántica propositiva del trabajo social frente a los desafios de la globalización. Revista de Trabajo Social, 71, 55-72.

Organización de Naciones Unidas (ONU) (s.f.). Carta de Derechos. New York: Autor.

Rivera de Alvarado, Carmen. (1986). Lucha y visión de Puerto Rico libre. Río Piedras, Puerto Rico: Fundación Carmen Rivera de Alvarado.

Torres, Carlos Alberto. (2001). Democracia, educación y multiculturalismo: Dilemas de la ciudadania en un mundo global, 1ra. Ed. Trad. Isabel Vericat y Berta Ruiz de la Concha. México: Editorial Siglo XXI. 
Van Soest, Dorothy. (1997). The global crisis of violence: Common problems, universal causes and share solutions. Washington, DC. NASW Press.

Vélez Restrepo, Olga Lucía. (2003) Reconfigurando el trabajo social: Perspectivas y tendencias contemporáneas. Argentina: Espacio Editorial.

Young, Iris Marion. (1990). Justice and the politics of difference. New Jersey, Estados Unidos: Princenton University Press. 
